\title{
Implications of automatic photon quenching on compact gamma-ray sources
}

\author{
M. Petropoulou and A. Mastichiadis \\ Department of Physics, University of Athens, Panepistimiopolis, 15783 Zografos, Greece \\ Received 22 February 2011 / Accepted 26 April 2011

\begin{abstract}
Aims. We investigate photon quenching in compact non-thermal sources. This involves photon-photon annihilation and lepton synchrotron radiation in a network that can become non-linear. As a result the $\gamma$-ray luminosity of a source cannot exceed a critical limit that depends only on the radius of the source and on the magnetic field.

Methods. We perform analytic and numerical calculations that verify previous results and extend them so that the basic properties of photon quenching are investigated.

Results. We apply the above to the $2006 \mathrm{TeV}$ observations of quasar 3C 279 and obtain the parameter space of allowed values for the radius of the emitting source, its magnetic field strength and the Doppler factor of the flow. We argue that the TeV observations favour either a modest Doppler factor and a low magnetic field or a high Doppler factor and a high magnetic field.
\end{abstract}

Key words. infrared: general - acceleration of particles - radiation mechnisms: non-thermal

\section{Introduction}

Astrophysical $\gamma$-ray sources are often subject to the so-called "compactness" problem: when the flux at longer wavelengths is combined with the dimensions of the source, which are inferred from light-crossing time arguments, a high optical thickness to photon-photon annihilation and effective absorption of $\gamma$-rays are implied.

The possibility that high-energy photons could pair-produce on soft target photons instead of escape in compact sources was first discussed by Jelley (1966), while the ratio of the luminosity to the size of the source, $L / R$, emerges as a determining factor of whether or not a high-energy photon will actually be absorbed (Herterich 1974). This was followed by work that took into account photon-photon annihilation not only as a sink of $\gamma$-rays, but also as a source of electron-positron pairs inside non-thermal compact sources (Bonometto \& Rees 1971; Guilbert et al. 1983; Kazanas 1984; Zdziarski \& Lightman 1985; Svensson 1987). The aim of these models was to calculate self-consistently the photon flux escaping from the sources by taking into account the energy redistribution caused by the $\gamma$-annihilation-induced pair cascades. The assumption was that high-energy particles or $\gamma$-rays were injected uniformly in a source that also contains soft photons, and the system was followed to its final steadystate through the solution of a set of kinetic equations describing the physical processes at work. Several groups have also developed numerical codes for the computation of time-dependent solutions to the kinetic equations taking photon-photon annihilation into account (Coppi 1992; Mastichiadis \& Kirk 1995; Stern et al. 1995; Böttcher \& Chiang 2002). These algorithms are commonly used in source modelling (Mastichiadis \& Kirk 1997; Kataoka et al. 2000; Konopelko et al. 2003; Katarzyński et al. 2005).

One of the assumptions of these models, as stated above, is the presence of soft photons in the source, which serve as targets for the $\gamma$-ray annihilation. A different approach has been recently presented by Stawarz \& Kirk (2007, henceforth SK). These authors focused on the non-linear effects induced by photonphoton annihilation and investigated the necessary conditions under which $\gamma$-ray photons can cause runaway pair production ${ }^{1}$. Stawarz \& Kirk (2007) showed that there is a limit to the $\gamma$-ray luminosity escaping from a source, which does not rely on the existing soft photon population but is instead a theoretical limit depending only on parameters such as the source size and its magnetic field strength. Violation of this limit leads to automatic quenching of the $\gamma$-rays. This involves a network of processes, namely photon-photon annihilation and lepton synchrotron radiation, which can become non-linear once certain criteria are satisfied. In this case electron-positron pairs grow spontaneously in the system and the "excessive" $\gamma$-rays are absorbed on the synchrotron photons emitted by the pairs. As a result the system reaches a final steady state where the $\gamma$-rays, soft photons, and electron-positron pairs have all reached equilibrium. Therefore this can occur even in the hypothetical case when there are no soft photons in the source, at least initially.

Indeed, photon quenching has some interesting implications for emission models of $\gamma$-rays because it gives a robust upper limit on the $\gamma$-ray luminosity. The aim of the present paper is to investigate the implications of this network for the high-energy compact astrophysical sources. In Sect. 2 we will expand the analytical approach of SK, who studied the dynamical system of photons and relativistic pairs using $\delta$-functions for the photonphoton annihilation cross section and for the synchrotron emissivity, while they treated synchrotron losses as catastrophic. In Sect. 3 we will use a numerical approach that will allow us to study the properties of quenching using the full cross section for $\gamma \gamma$ absorption and the full synchrotron emissivity. As an example, in Sect. 4 the above will be applied to the 2006 MAGIC TeV

\footnotetext{
1 We note that in the same framework an analogous study where ultrarelativistic protons are the primary injected particles has earlier been presented by Kirk \& Mastichiadis (1992).
} 
observations of quasar 3C 279 to extract a parameter space of allowed values for the source parameters, i.e., the radius $R$ and the magnetic field strength $B$, as well as for the Doppler factor $\delta$ of the flow. Finally in Sect. 5 we conclude and give a brief discussion of the main points of the present work.

\section{Analytical approach}

\subsection{First principles}

We begin by assuming a spherical source of radius $R$ containing a tangled magnetic field of strength $B$. We also assume that monoenergetic $\gamma$-rays of energy $\epsilon_{\gamma}$ (in units of $m_{\mathrm{e}} c^{2}$ ) are uniformly produced by some unspecified mechanism throughout the volume of the source. If these are injected with a luminosity $L_{\gamma}^{\text {inj }}$, one can define the injected $\gamma$-ray compactness as

$\ell_{\gamma}^{\mathrm{inj}}=\frac{L_{\gamma}^{\mathrm{inj}} \sigma_{T}}{4 \pi R m_{\mathrm{e}} c^{3}}$,

where $\sigma_{T}$ is the Thomson cross section. Without any substantial soft photon population inside the source, the $\gamma$-rays will escape without any attenuation in one crossing time. However, as SK showed, the injected $\gamma$-ray compactness cannot become arbitrarily high because if a critical value is reached, the following loop starts operating:

1. gamma-rays pair-produce on soft photons, which can be arbitrarily low inside the source;

2. the produced electron-positron pairs cool by emitting synchrotron photons, thus acting as a source of soft photons;

3 . the soft photons serve as targets for more $\gamma \gamma$ interactions.

There are two conditions that should be satisfied simultaneously for this network to occur: the first, which is a feedback condition, requires that the synchrotron photons emitted from the pairs have sufficient energy to pair-produce on the $\gamma$-rays. By making suitable simplifying assumptions, one can derive an analytic relation for it - see also SK. Thus, combining (1) the threshold condition for $\gamma \gamma$-absorption $\epsilon_{\gamma} \epsilon_{0}=2$; (2) the fact that there is equipartition of energy among the created electron-positron pairs $\gamma_{\mathrm{p}}=\gamma_{\mathrm{e}}=\gamma=\epsilon_{\gamma} / 2$; and (3) the assumption that the required soft photons of energy $\epsilon_{0}$ are the synchrotron photons that the electrons/positrons radiate, i.e., $\epsilon_{0}=b \gamma^{2}$ where $b=B / B_{\text {crit }}$ and $B_{\text {crit }}=\left(m_{\mathrm{e}}^{2} c^{3}\right) /(e \hbar) \simeq 4.4 \times 10^{13} \mathrm{G}$ is the critical value of the magnetic field, one derives the minimum value of the magnetic field required for quenching to become relevant

$B_{\mathrm{q}}=8 \epsilon_{\gamma}^{-3} B_{\text {crit }}$.

Thus for $B \geq B_{\mathrm{q}}$ the feedback criterion is satisfied. Note that this is a condition that only contains the emitted $\gamma$-ray energy and the magnetic field strength; moreover, it can easily be satisfied, at least if one is to use the values inferred from typical modelling of the sources (Böttcher 2007; Böttcher et al. 2009).

Along with the feedback criterion, a second criterion must be imposed for the quenching to be fully operative. A simple way to see this is with the following consideration. Assume that the $\gamma$-rays pair-produce on some soft photon and that the created electron-positron pairs cool by emitting synchrotron photons. Because an electron emits several such photons before cooling the critical condition occurs if the number density of the $\gamma$-rays is such that at least one of the synchrotron photons pair-produces on a $\gamma$-ray instead of escaping from the source. Thus the condition for criticality can be written as

$\mathcal{N}_{\mathrm{s}}\left(\epsilon_{\gamma} / 2\right) n\left(\epsilon_{\gamma}\right) \sigma_{\gamma \gamma} R \geq 1$, where $n\left(\epsilon_{\gamma}\right)$ is the number density of $\gamma$-rays, $\sigma_{\gamma \gamma}$ is the cross section for $\gamma \gamma$ interactions and $\mathcal{N}_{\mathrm{s}}(\gamma)$ is the number of synchrotron photons emitted by an electron with Lorentz factor $\gamma$ before it cools. Assuming that the pair-producing collisions occur close to threshold, approximating the cross section there by $\sigma_{\gamma \gamma} \simeq \sigma_{\mathrm{T}} / 3$ and using $\mathcal{N}_{\mathrm{s}}(\gamma) \simeq \frac{\gamma}{b \gamma^{2}}$ and $n\left(\epsilon_{\gamma}\right)=\frac{L_{\gamma}^{\mathrm{inj}}}{V \epsilon_{\gamma} m_{\mathrm{e}} c^{2}} \frac{R}{c}$, the critical condition can be written as

$$
\epsilon_{\gamma} \ell_{\gamma}^{\mathrm{inj}} \geq 4
$$

where the feedback condition (2) and Eq. (1) were also used. Taken as equalities, relations (2) and (4) define the marginal stability criterion, which essentially is a condition for the $\gamma$-ray luminosity.

\subsection{Kinetic equations}

We begin by writing the kinetic equations that describe the distributions of $\gamma$-ray photons, soft photons, and electrons in the source. These are respectively

$$
\begin{aligned}
\frac{\partial n\left(\epsilon_{\gamma}, \tau\right)}{\partial \tau}+n & =Q_{\mathrm{inj}}^{\gamma}+\mathcal{L}_{\gamma \gamma}^{\gamma} \\
\frac{\partial n_{0}(x, \tau)}{\partial \tau}+n_{0} & =Q_{\mathrm{syn}}^{\mathrm{s}}
\end{aligned}
$$

and

$$
\frac{\partial n_{\mathrm{e}}(\gamma, \tau)}{\partial \tau}=\mathcal{Q}_{\gamma \gamma}^{\mathrm{e}}+\mathcal{L}_{\mathrm{syn}}^{\mathrm{e}}
$$

where $n, n_{0}$ and, $n_{\mathrm{e}}$ are the differential $\gamma$-ray, soft photon, and electron number densities, respectively, and $\epsilon_{\gamma}, x$, and $\gamma$ are the corresponding energies normalized in $m_{\mathrm{e}} c^{2}$ units. The densities refer to the number of particles contained in a volume element $\sigma_{\mathrm{T}} R$. In other words, if $\hat{n}_{i}$ expresses the number of particles of species $i$ per ergs per $\mathrm{cm}^{3}$, then $n_{i}=\hat{n}_{i}\left(\sigma_{\mathrm{T}} R\right)\left(m_{\mathrm{e}} c^{2}\right)$. Time is normalized with respect to the photon crossing/escape time from the source, $t_{\mathrm{cr}}=R / c$. Thus, $\tau$, which appears in the kinetic equations, is dimensionless and equals $\tau=\frac{c t}{R}$. The operators $Q$ and $\mathcal{L}$ denote injection and losses, respectively. The only processes that we take into account are $\gamma \gamma$ annihilation and synchrotron cooling of the produced pairs. The synchrotron emissivity is approximated by a $\delta$-function, i.e., $j_{\mathrm{s}}(x)=j_{0} \delta\left(x-\epsilon_{0}\right)$, where $\epsilon_{0}=b \gamma^{2}$ is the synchrotron critical energy. In all cases we will assume a monoenergetic $\gamma$-ray injection at energy $\epsilon_{\gamma}$.

\subsection{1. $\delta$-function approximation for the $\sigma_{\gamma \gamma}$ cross section}

First we will examine the stability of the system using the simplest form for the photon-photon annihilation cross section (Zdziarski \& Lightman 1985):

$\sigma_{\gamma \gamma}(x)=\frac{\sigma_{\mathrm{T}}}{3} x \delta\left(x-\frac{2}{\epsilon_{\gamma}}\right)$,

where $x, \epsilon_{\gamma}$ are the energies of the soft and $\gamma$-ray photons, respectively. The particle injection and loss operators take the 
following forms:

$$
\begin{aligned}
Q_{\text {inj }}^{\gamma} & =\frac{3 l_{\gamma}^{\text {inj }}}{\epsilon_{\gamma}^{2}} \\
Q_{\text {syn }}^{\mathrm{s}} & =\frac{2}{3} l_{B} b^{-3 / 2} x^{-1 / 2} n_{\mathrm{e}}(\sqrt{x / b}, \tau) \\
\mathcal{L}_{\gamma \gamma}^{\gamma} & =-\frac{2}{3} \frac{n_{0}\left(2 / \epsilon_{\gamma}\right) n\left(\epsilon_{\gamma}\right)}{\epsilon_{\gamma}} \delta\left(\epsilon_{\gamma}-2 \gamma\right) \\
\mathcal{L}_{\text {syn }}^{\mathrm{e}} & =+\frac{4}{3} l_{B} \frac{\partial}{\partial \gamma}\left(\gamma^{2} n_{\mathrm{e}}\right)
\end{aligned}
$$

and

$$
Q_{\gamma \gamma}^{\mathrm{e}}=-4 \mathcal{L}_{\gamma \gamma}^{\gamma}
$$

because each photon-photon annihilation results in a pair of leptons, each one with approximately half of the initial $\gamma$-ray energy. In the above equations the "magnetic compactness" was introduced

$l_{B}=\left(\frac{U_{B}}{m_{\mathrm{e}} c^{2}}\right) \sigma_{\mathrm{T}} R$.

The system of Eqs. (5)-(7) can now be written as

$$
\begin{aligned}
\frac{\partial n\left(\epsilon_{\gamma}\right)}{\partial \tau}= & -n\left(\epsilon_{\gamma}\right)-\frac{2}{3} \frac{n_{0}\left(2 / \epsilon_{\gamma}\right) n\left(\epsilon_{\gamma}\right)}{\epsilon_{\gamma}} \delta\left(\epsilon_{\gamma}-2 \gamma\right)+\frac{3 l_{\gamma}^{\mathrm{inj}}}{\epsilon_{\gamma}^{2}} \\
\frac{\partial n_{0}(x)}{\partial \tau}= & -n_{0}(x)+\frac{2}{3} l_{B} b^{-3 / 2} x^{-1 / 2} n_{\mathrm{e}}(\sqrt{x / b}) \\
\frac{\partial n_{\mathrm{e}}(\gamma)}{\partial \tau}= & +\frac{4}{3} \frac{n_{0}\left(2 / \epsilon_{\gamma}\right) n\left(\epsilon_{\gamma}\right)}{\epsilon_{\gamma}} \delta\left(\gamma-\epsilon_{\gamma} / 2\right) \\
& +\frac{4}{3} l_{B} \frac{\partial}{\partial \gamma}\left(\gamma^{2} n_{\mathrm{e}}(\gamma)\right) .
\end{aligned}
$$

In this section we will examine the stability of the trivial stationary solution of the system (15):

$\bar{n}=3 l_{\gamma}^{\mathrm{inj}} / \epsilon_{\gamma}^{2}, \bar{n}_{0}=0, \bar{n}_{\mathrm{e}}=0$,

which corresponds to the free propagation of $\gamma$-ray photons through the source. To investigate the stability of the system, we assume that initially arbitrarily small perturbations of the soft photon $n_{0}^{\prime}$ and electron $n_{\mathrm{e}}^{\prime}$ densities are present, which lead to the perturbation of the $\gamma$-ray photon density $n^{\prime}$. After linearization, the system (15) becomes

$$
\begin{aligned}
& \frac{\partial n^{\prime}}{\partial \tau}=-n^{\prime}-\frac{2}{3} \frac{n_{0}^{\prime} \bar{n}}{\epsilon_{\gamma}} \delta\left(\epsilon_{\gamma}-2 \gamma\right) \\
& \frac{\partial n_{0}^{\prime}}{\partial \tau}=-n_{0}^{\prime}+\frac{2}{3} l_{B} b^{-3 / 2} x^{-1 / 2} n_{\mathrm{e}}^{\prime} \\
& \frac{\partial n_{\mathrm{e}}^{\prime}}{\partial \tau}=+\frac{4}{3} \frac{n_{0}^{\prime} \bar{n}}{\epsilon_{\gamma}} \delta\left(\gamma-\epsilon_{\gamma} / 2\right)+\frac{4}{3} l_{B} \frac{\partial}{\partial \gamma}\left(\gamma^{2} n_{\mathrm{e}}^{\prime}\right) .
\end{aligned}
$$

The stability analysis of the system above can be simplified when one works with the Laplace transformed number densities:

$n_{0}^{\prime}(x, \tau) \rightarrow n_{0}^{\prime}(x, s)=\int_{0}^{\infty} \mathrm{d} \tau n_{0}^{\prime}(x, \tau) \mathrm{e}^{-s \tau}$ with $s$ being the solution to the eigenvalue problem:

$$
\begin{aligned}
(s+1) n^{\prime}\left(\epsilon_{\gamma}, s\right)= & -\frac{2}{3} \frac{n_{0}^{\prime}\left(2 / \epsilon_{\gamma}, s\right) \bar{n}}{\epsilon_{\gamma}} \\
(s+1) n_{0}^{\prime}(x, s)= & \frac{2}{3} l_{B} b^{-3 / 2} x^{-1 / 2} n_{\mathrm{e}}^{\prime}(\sqrt{x / b}, s) \\
s n_{\mathrm{e}}^{\prime}(\gamma, s)= & +\frac{4}{3} \frac{n_{0}^{\prime}\left(2 / \epsilon_{\gamma}, s\right) \bar{n}}{\epsilon_{\gamma}} \delta\left(\gamma-\epsilon_{\gamma} / 2\right) \\
& +\frac{4}{3} l_{B} \frac{\partial}{\partial \gamma}\left(\gamma^{2} n_{\mathrm{e}}^{\prime}(\gamma, s)\right) .
\end{aligned}
$$

For large $\tau$ both the soft photon and electron distributions behave as $e^{s \tau}$. If $s=-1$, then $n_{0}^{\prime}=n_{\mathrm{e}}^{\prime}=0$ and the $\gamma$-ray photons freely escape from the source. If $s>0$, one finds solutions that grow with elapsing time. The marginally stable solution, which is obtained when one sets $s=0$, will be examined below.

Combining Eqs. (19)-(21) leads to an ordinary differential equation for the perturbed electron distribution with the following solution:

$\gamma^{2} n_{\mathrm{e}}^{\prime}(\gamma)=C-\frac{2 \bar{n} n_{\mathrm{e}}^{\prime}\left(\gamma_{\mathrm{cr}}\right)}{3 b^{3 / 2} \sqrt{2 \epsilon_{\gamma}}}$,

where $\gamma_{\mathrm{cr}}=\sqrt{\frac{2}{b \epsilon_{\gamma}}}$. Setting $\gamma=\gamma_{\mathrm{cr}}$ in the above equation one finds

$n_{\mathrm{e}}^{\prime}(\gamma)=C\left(\frac{\gamma_{\mathrm{cr}}}{\gamma}\right)^{2} \frac{1}{\gamma_{\mathrm{cr}}^{2}+\frac{2 \bar{n}}{3 b^{3 / 2} \sqrt{2 \epsilon_{\gamma}}}}$.

Then the perturbed photon distribution is found using Eq. (20).

As already mentioned in Sect. 2.1, the $\gamma$-ray compactness cannot become arbitrarily high but instead saturates at a critical value $l_{\gamma, \mathrm{cr}}^{\mathrm{inj}}$. When $l_{\gamma}^{\text {inj }} \ll l_{\gamma, \mathrm{cr}}^{\text {inj }}$ only a negligible fraction of the hard photon luminosity will be absorbed, i.e., $l_{\gamma}^{h^{\prime}} \rightarrow 0$, or equivalently $l_{\gamma}^{h} \propto l_{\gamma}^{\text {inj }}$. We call critical the injected luminosity required for $l_{\gamma}^{h^{\prime}}=l_{\gamma}^{s^{\prime}}$ to occur. After this point any further increase of the injected luminosity will be seen as an increase of the luminosity in soft photons. Using Eqs. (19), (20), and (23) one derives

$\epsilon_{\gamma}^{2} n^{\prime}\left(\epsilon_{\gamma} ; l_{\gamma, \mathrm{cr}}^{\mathrm{inj}}\right)=\int^{x_{\max }} \mathrm{d} x x n_{0}^{\prime}\left(x ; l_{\gamma, \mathrm{cr}}^{\mathrm{inj}}\right)$

where $x_{\max }=b \epsilon_{\gamma}^{2} / 4$. Solving the above equation with respect to $l_{\gamma, \mathrm{cr}}^{\mathrm{inj}}$, we find

$l_{\gamma, \mathrm{cr}}^{\mathrm{inj}}=\sqrt{2 b \epsilon_{\gamma}}$.

To compare our results with the value $l_{\mathrm{cr}}^{\mathrm{SK}}=3$ found by SK, who have used catastrophic synchrotron losses, we have to take the following steps:

1. the case of synchrotron cooling degenerates to the "catastrophic losses case" when the threshold energy for absorption $2 / \epsilon_{\gamma}$ equals the maximum soft photon energy $x_{\max }$;

2. in SK the volume element is defined as $V=\pi R^{3}$, whereas we used $V=4 / 3 \pi R^{3}$. Thus, the replacement $l_{\mathrm{cr}}^{\mathrm{SK}} \rightarrow \frac{4}{3} l_{\mathrm{cr}}^{\mathrm{SK}}$ should be made;

3. finally, in SK the luminosity compactness is not given by the conventional definition, which we adopted, but is multiplied by the energy $\epsilon_{\gamma}$. 


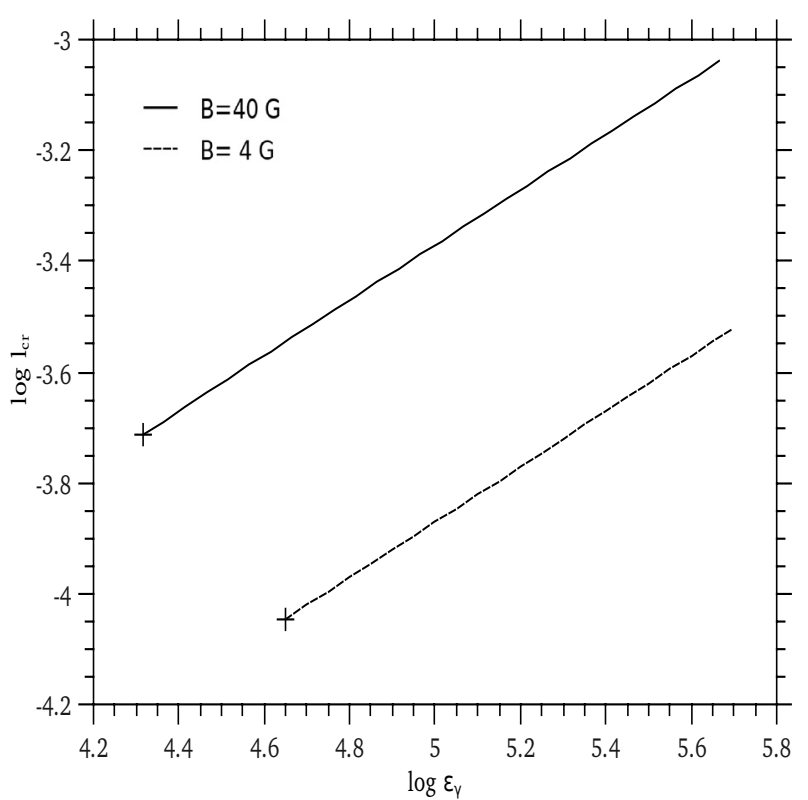

Fig. 1. Log-log plot of the critical luminosity compactness $l_{\gamma, \text { cr }}^{\text {inj }}$ as a function of the injected $\gamma$-ray photon energy $\epsilon_{\gamma}$, when the $\delta$-function approximation for the cross section $\sigma_{\gamma \gamma}$ was used for two values of the magnetic field strength: $B=40 \mathrm{G}$ (solid line) and $B=4 \mathrm{G}$ (dashed line). The values calculated by SK using only catastrophic losses are marked with crosses.

Thus, our result should be compared to $\frac{4 l_{\mathrm{cr}}^{\mathrm{SK}}}{3 \epsilon_{\gamma}}$. The critical compactness as a function of $\epsilon_{\gamma}$ for two values of the magnetic field is shown in Fig. 1. The two crosses mark the values of SK, which completely agree with ours in the limit of catastrophic losses.

We note also that Eq. (4), which was obtained by making robust calculations, also agrees with Eq. (25) in the limit of catastrophic losses.

\subsubsection{Step function approximation for the $\sigma_{\gamma \gamma}$ cross section}

In this paragraph we analytically find the critical luminosity compactness after adopting a more realistic expression for the annihilation cross section:

$\sigma_{\gamma \gamma}=\sigma_{\mathrm{T}} \sigma_{0} \frac{\Theta\left(x \epsilon_{\gamma}-2\right)}{x \epsilon_{\gamma}}$

where the normalization used is $\sigma_{0}=4 / 3$. The initial Eqs. (5)(7) keep the same form. Only the operator of photon losses becomes

$\mathcal{L}_{\gamma \gamma}^{\gamma}=n(2 \gamma) \int \mathrm{d} x \sigma_{0} \frac{\Theta\left(x \epsilon_{\gamma}-2\right)}{x \epsilon_{\gamma}} n_{0}^{\prime}(x)$.

Following the same steps as those described in Sect. 2.2.1, we find for $s=0$

$\gamma^{2} n_{\mathrm{e}}^{\prime}(\gamma)=C-A \int_{\gamma_{\mathrm{cr}}}^{\gamma_{\max }} \mathrm{d} \gamma \gamma^{-2} n_{\mathrm{e}}^{\prime}(\gamma)$

where $A=\frac{2 \bar{n} \sigma_{0}}{b^{2} \epsilon_{\gamma}}$ and $\gamma_{\max }=\epsilon_{\gamma} / 2$. Equation (28) can be solved iteratively when writing $n_{\mathrm{e}}^{\prime}(\gamma)$ as a sum of approximations, i.e., $n_{\mathrm{e}}^{\prime}(\gamma)=n^{(0)}+n^{(1)}+\ldots$. Assuming for the first approximation the form $n^{(0)}=C \gamma^{-2}$, we find the other terms of the series expansion:

$n^{(1)}=C \gamma^{-2} \lambda$

$n^{(2)}=C \gamma^{-2} \lambda^{2}$,

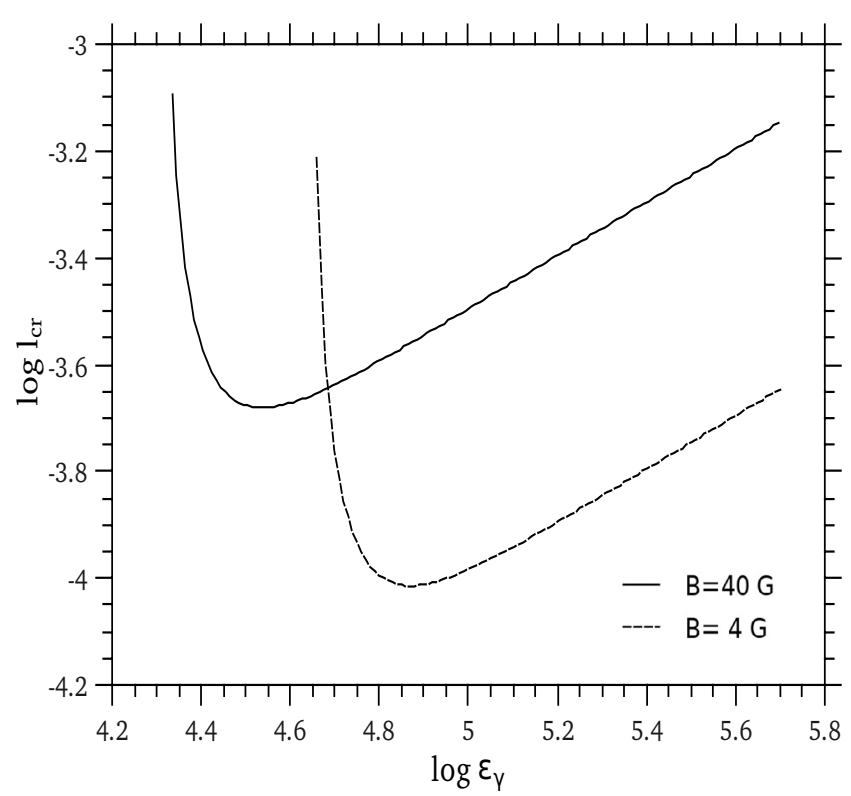

Fig. 2. Log-log plot of the critical luminosity compactness $l_{\gamma, \text { cr }}^{\text {inj as a }}$ function of the injected $\gamma$-ray photon energy $\epsilon_{\gamma}$, when the $\Theta$-function approximation for the cross section $\sigma_{\gamma \gamma}$ was used for two values of the magnetic field strength: $B=40 \mathrm{G}$ (solid line) and $B=4 \mathrm{G}$ (dashed line).

where $\lambda=\frac{A}{3}\left(\frac{1}{\gamma_{\max }^{3}}-\frac{1}{\gamma_{\text {cr }}^{3}}\right)$ and $|\lambda|<1$ for typical values of the parameters. Thus, the series of approximations converges and $n_{\mathrm{e}}^{\prime}$ can be found in closed form:

$n_{\mathrm{e}}^{\prime}(\gamma)=\frac{C}{\gamma^{2}} \sum_{n=1}^{\infty} \lambda^{n-1}=\frac{C}{\gamma^{2}(1-\lambda)}$.

The luminosity in soft and hard photons can then be found

$l_{\gamma}^{s^{\prime}}=\frac{4 l_{B}}{3} \frac{C \epsilon_{\gamma}}{2(1-\lambda)}$

$l_{\gamma}^{h^{\prime}}=\frac{2 l_{B} C \epsilon_{\gamma}^{2}}{3} \frac{|\lambda|}{1-\lambda}$.

The critical $\gamma$-ray compactness is given by

$l_{\gamma, \mathrm{cr}}^{\mathrm{inj}}=\frac{b^{2} \epsilon_{\gamma}^{2}}{2 \sigma_{0}}\left[\left(\frac{b \epsilon_{\gamma}}{2}\right)^{3 / 2}-\frac{8}{\epsilon_{\gamma}^{3}}\right]^{-1}$.

Figure 2 shows $l_{\gamma, \text { cr }}^{\text {inj }}$ as a function of $\epsilon_{\gamma}$ for two values of the magnetic field. When the threshold energy for absorption $2 / \epsilon_{\gamma}$ is much lower than the maximum energy of the soft synchrotron photons $b \epsilon_{\gamma}^{2} / 4$, the critical compactness as a function of $\epsilon_{\gamma}$ behaves as for the $\delta$-function cross section. However, when the two energies become comparable, $l_{\gamma, \mathrm{in}}^{\text {in }}$ abruptly increases, because the number of soft photons serving as targets for the absorption significantly decreases. This effect can be seen in Fig. 2 as a change in the curvature of the curves.

\section{Numerical approach}

To numerically investigate the properties of quenching, one needs to solve again the system of Eqs. (5)-(7) augmented to include more physical processes. As in the numerical code, there 
is no need to treat the time-evolution of soft photons and $\gamma$-rays through separate equations, the system can be written:

$$
\frac{\partial n_{\mathrm{e}}(\gamma, t)}{\partial t}+\frac{n_{\mathrm{e}}}{t_{\mathrm{e}, \mathrm{esc}}}=Q_{\gamma \gamma}^{\mathrm{e}}+\mathcal{L}_{\mathrm{syn}}^{\mathrm{e}}+\mathcal{L}_{\mathrm{ics}}^{\mathrm{e}}
$$

and

$$
\frac{\partial n_{\gamma}(x, t)}{\partial t}+\frac{n_{\gamma}}{t_{\gamma, \mathrm{esc}}}=\mathcal{L}_{\gamma \gamma}^{\gamma}+Q_{\mathrm{syn}}^{\gamma}+Q_{\mathrm{ics}}^{\gamma}+\mathcal{L}_{\mathrm{ssa}}^{\gamma}+Q_{\mathrm{inj}}^{\gamma},
$$

where $n_{\mathrm{e}}$ and $n_{\gamma}$ are the differential electron and photon number density, respectively, normalized as in Sect. 2. Here we considered the following processes: (i) photon-photon pair production, which acts as a source term for electrons $\left(Q_{\gamma \gamma}^{\mathrm{e}}\right)$ and a sink term for photons $\left(\mathcal{L}_{\gamma \gamma}^{\gamma}\right)$; (ii) synchrotron radiation, which acts as a loss term for electrons $\left(\mathcal{L}_{\text {syn }}^{\mathrm{e}}\right)$ and a source term for photons $\left(Q_{\text {syn }}^{\gamma}\right)$; (iii) synchrotron self-absorption, which acts as a loss term for photons $\left(\mathcal{L}_{\mathrm{ssa}}^{\gamma}\right)$; and (iv) inverse Compton scattering, which acts as a loss term for electrons $\left(\mathcal{L}_{\text {ics }}^{\text {e }}\right)$ and a source term for photons $\left(Q_{\text {ics }}^{\gamma}\right)$. In addition to the above, we assume that $\gamma$-rays are injected into the source through the term $\left(Q_{\text {inj }}^{\gamma}\right)$. The functional forms of the various rates have been presented elsewhere - Mastichiadis \& Kirk (1997) and Petropoulou \& Mastichiadis (2009). The photons are assumed to escape the source in one crossing time, therefore $t_{\gamma, \text { esc }}=R / c$.

The parameters of the problem are completely specified once the values of radius $R$ and of the magnetic field $B$ are set and the injection rate $Q_{\text {inj }}^{\gamma}$ is specified. Because $Q_{\text {inj }}^{\gamma}$ is readily related to the injected luminosity $L_{\gamma}^{\mathrm{inj}}$ through the relation

$L_{\gamma}^{\mathrm{inj}}=\left(m_{\mathrm{e}} c^{2}\right)^{2} V \int \mathrm{d} x \times Q_{\mathrm{inj}}^{\gamma}$,

where $V$ is the volume of the source, the injection rate is specified once the injection compactness $\ell_{\gamma}^{\text {inj }}$ and the functional dependence of $Q_{\text {inj }}^{\gamma}$ on $\epsilon_{\gamma}$ is set. The electron physical escape timescale from the source $t_{\mathrm{e}, \text { esc }}$ is another free parameter which, however, is not important in our case. Thus, we will fix it at value $t_{\mathrm{e}, \mathrm{esc}}=t_{\gamma, \mathrm{esc}}=R / c$. The final settings are the initial conditions for the electron and photon number densities. Because we are investigating the spontaneous growth of pairs and synchrotron photons, we assume that at $t=0$ there are no electrons or photons in the source, so we set $n_{\mathrm{e}}(\gamma, 0)=n_{\gamma}(x, 0)=0$.

\subsection{Monoenergetic $\gamma$-ray injection}

As a first example we study the case where the $\gamma$-rays injected are monoenergetic at an energy $\epsilon_{\gamma}$. The expected trivial solution of the kinetic equations is that because there are no soft photons in the source, the injected $\gamma$-rays will freely escape and their spectrum upon escape will simply be their production one. Electrons and photons at energies different from $\epsilon_{\gamma}$ will remain at their initial values, i.e., $n_{\mathrm{e}}(\gamma, t)=n_{\gamma}(x, t)=0$.

Before numerically examining the stability of the above system using the full expressions for the photon-photon annihilation cross section and the emissivities, we perform a test by comparing the analytical result given by Eq. (34) with the respective numerical. The two solutions completely agree as shown in Fig. 3. In the same figure we also plot the critical $\gamma$-ray compactness found numerically when all quantities used were given by their full expressions. The qualitative behaviour of the full problem solution is quite well reproduced by the analytical one obtained while using the $\Theta$ - and $\delta$-approximations for the cross

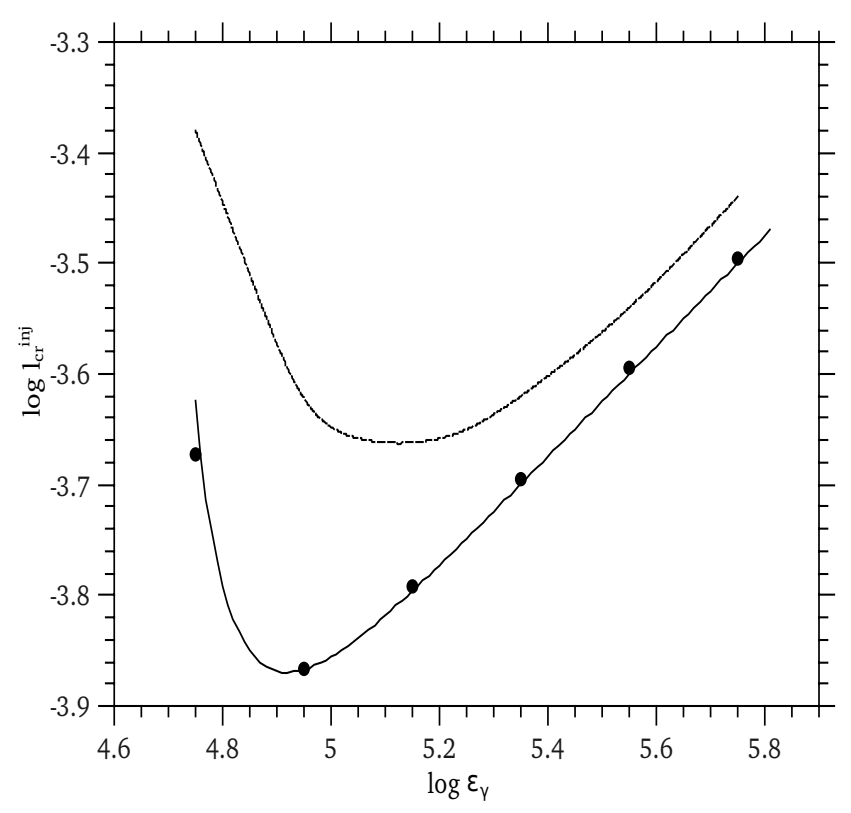

Fig. 3. Critical $\gamma$-ray compactness as a function of $\epsilon_{\gamma}$ when (i) $\sigma_{\gamma \gamma}$ and synchrotron emissivity are approximated by a $\Theta$ - and a $\delta$-function respectively; and (ii) when for both quantities the full expressions are used. For the first case the numerical result (points) and the analytical one given by Eq. (34) (solid line) are shown. The full problem can only be treated numerically and the corresponding result is shown with a dashed line. The size of the source is $R=3 \times 10^{16} \mathrm{~cm}$ and the magnetic field strength $B=3.57 \mathrm{G}$.

section and synchrotron emissivity respectively. This allows us to use the analytical expression given by Eq. (34) for the full problem as well, when investigating the properties of quenching at least qualitatively. From this figure it becomes apparent that $\gamma$-ray quenching can affect all photons with energies that satisfy the feedback condition. Starting around this energy and moving progressively to higher ones, we find that the critical compactness first sharply decreases until it reaches a minimum and then starts increasing again, as $l_{\gamma, \mathrm{cr}}^{\mathrm{inj}} \propto \epsilon_{\gamma}^{1 / 2}$ asymptotically. The exact value of the critical compactness also depends on the strength of the magnetic field. For a higher $B$-field the required minimum energy $\epsilon_{\gamma}$ decreases, which can be easily deduced by Eq. (2). Note also that because of the marginal relation (34), the required critical injected compactness becomes higher. Thus, by increasing $B$, the corresponding curve is shifted towards lower $\gamma$-ray energies and higher critical compactnesses, keeping its shape (see e.g. Fig. 2).

Figure 4 depicts the results of a series of runs where we kept $\epsilon_{\gamma}$ constant and varied $\ell_{\gamma}^{\text {inj }}$. For sufficiently low values of the latter parameter, all $\gamma$-rays indeed escape from the system without any impedance. If we call $\ell_{\gamma}^{h}$ the compactness of the outgoing radiation at energy $\epsilon_{\gamma}$, then obviously, in this case, $\ell_{\gamma}^{\text {out }}=\ell_{\gamma}^{h}=\ell_{\gamma}^{\text {inj }}$, where $\ell_{\gamma}^{\text {out }}$ is the compactness of the outgoing luminosity. However, above a critical value of $\ell_{\gamma}^{\text {inj }}$, which we will call $\ell_{\gamma, \mathrm{cr}}^{\mathrm{inj}}$, the loop described above starts operating, transferring the $\gamma$-ray luminosity to softer radiation. Thus, for $\ell_{\gamma}^{\text {inj }}>\ell_{\gamma, \mathrm{cr}}^{\mathrm{inj}}$, we get $\ell_{\gamma}^{\text {out }}=\ell_{\gamma}^{\mathrm{s}}+\ell_{\gamma}^{h}=\ell_{\gamma}^{\mathrm{s}}+\ell_{\gamma, \mathrm{cr}}^{\mathrm{inj}}$ because $\ell_{\gamma}^{h}$ saturates - by $\ell_{\gamma}^{\mathrm{s}}$ we denote the compactness of the reprocessed luminosity, which appears at lower energies. This can be seen in Fig. 4, where the dashed line curve represents $\ell_{\gamma}^{h}$, the dotted line $\ell_{\gamma}^{\mathrm{s}}$ and the full line $\ell_{\gamma}^{\text {out }}$, which is always equal to $\ell_{\gamma}^{\text {inj }}$ because there are no sinks 


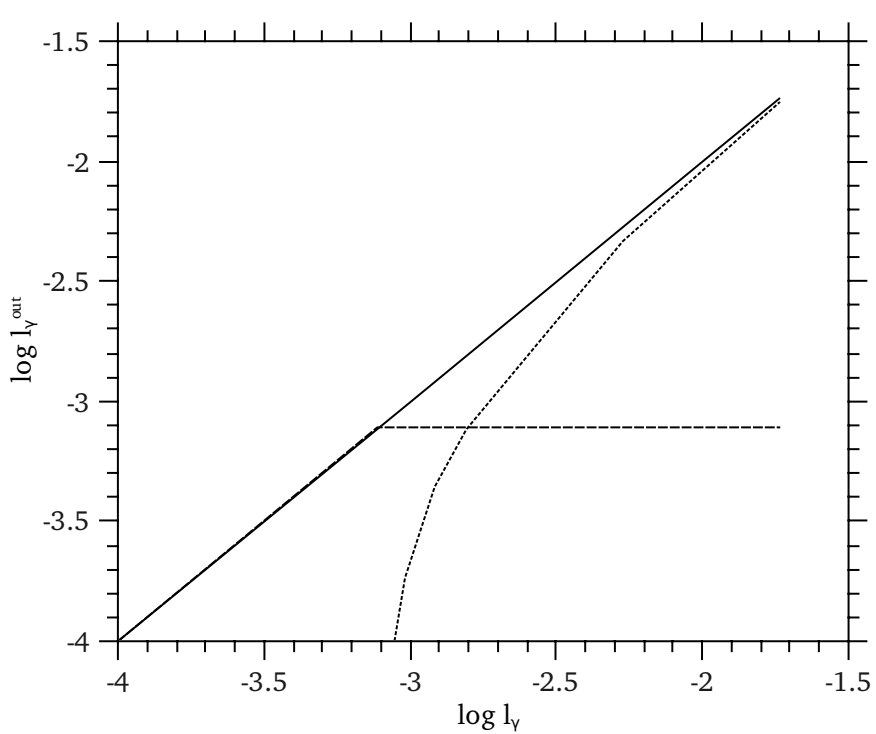

Fig. 4. Plot of the compactness of emerging hard $\ell_{\gamma}^{h}$ (dashed), soft $\ell_{\gamma}^{\mathrm{s}}$ (dotted), and total radiated luminosity $\ell_{\gamma}^{\text {out }}$ (full line) as a function of the injected compactness $\ell_{\gamma}^{\text {inj }}$. The $\gamma$-rays are assumed to be monoenergetic, injected at energy $\epsilon_{\gamma}=2.3 \times 10^{4}$. The size of the source is $R=3 \times$ $10^{16} \mathrm{~cm}$, while the magnetic field strength is $B=40 \mathrm{G}$. The emerging soft radiation is a result of the self-quenching of the system.

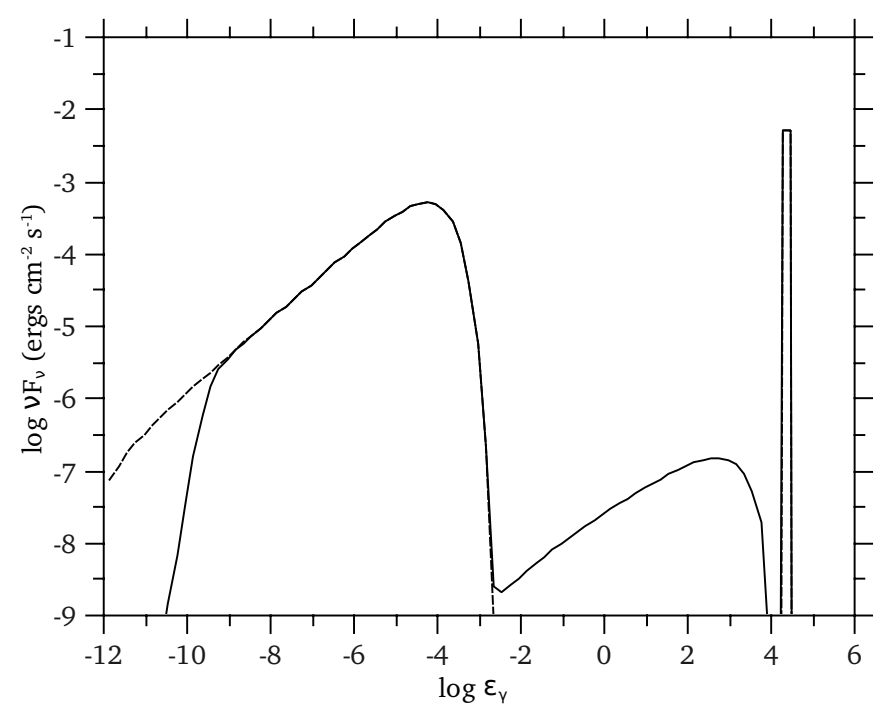

Fig. 5. Multiwavelength spectrum for a choice of $\ell_{\gamma}^{\text {inj }}$ such that $\ell_{\gamma}^{\mathrm{s}}=\ell_{\gamma}^{h}$. The other parameters are the same as in Fig. 4. Full line corresponds to the case where all relevant radiative processes were taken into account, while the dashed line corresponds to the case where only the two "core" processes were considered, i.e., synchrotron and $\gamma \gamma$ interactions.

of photons present. For the particular example shown in Fig. 4, the values of the other parameters used are, $R=3 \times 10^{16} \mathrm{~cm}$, $B=40 \mathrm{G}$ and $\epsilon_{\gamma}=2.3 \times 10^{4}$.

Figure 5 shows the multiwavelength (MW) spectrum for the particular value of $\ell_{\gamma}^{\text {inj }}$, which results in equipartition between soft and hard luminosities, i.e., $\ell_{\gamma}^{\mathrm{s}}=\ell_{\gamma}^{h}$. While the hard luminosity is simply the escaping monoenergetic radiation at $\epsilon_{\gamma}$, the soft spectrum mainly consists of the synchrotron component produced from the secondary pairs, which were injected at energies $\gamma_{\mathrm{p}}=\gamma_{\mathrm{e}}=\epsilon_{\gamma} / 2$. The peak of this component is at $\epsilon_{\mathrm{pk}}^{s}=b \gamma^{2}$, while it has a spectral index of $-1 / 2$ (or $+1 / 2$ in

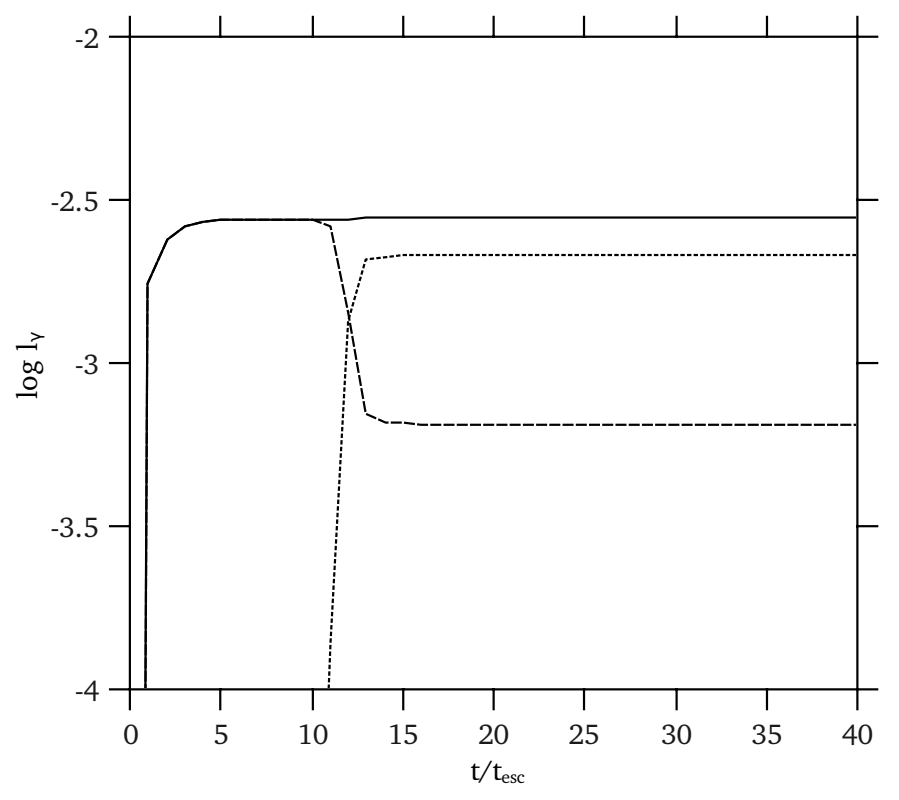

Fig. 6. Time evolution of the soft $l_{\gamma}^{s}$ (dotted line), hard $l_{\gamma}^{h}$ (dashed line) and total $l_{\gamma}$ (solid line) compactness for the same set of parameters as in Fig. 5.

$v F_{v}$ units) as a result of the electron cooling to a $\gamma^{-2}$ distribution below the energy of injection. There is also a synchrotron self-Compton (ssc) component, which is greatly suppressed by Klein-Nishina effects. As can be seen from the figure, the corrections introduced from the inclusion of inverse Compton scattering and synchrotron self-absorption are negligible, at least for our set of parameters.

Figure 6 shows the time evolution of the different luminosity compactnesses for the example case presented in Fig. 5. Although the dynamical, non-linear system of equations describing the system (see for example system of Eq. (15)) is of third order, which in principle can lead to unstable solutions or/and "chaotic" behaviour (SK), the system quickly reaches a stable state after a few crossing times. Figure 6 shows that although initially absent, a soft photon population builds up, which leads to a decrease of $l_{\gamma}^{h}$, because $\gamma$-rays are being absorbed by the soft photons.

\subsection{Power-law injection}

As a next step we investigate the effects of quenching for a power-law injection of $\gamma$-rays, i.e., when the injection rate can be written as

$Q_{\gamma}=Q_{0} \epsilon_{\gamma}^{-\alpha} \Theta\left(\epsilon_{\gamma}-\epsilon_{\gamma \min }\right) \Theta\left(\epsilon_{\gamma \max }-\epsilon_{\gamma}\right)$,

where $Q_{0}$ is a normalization constant depending on the value of $\alpha$, the low- and high- energy cutoffs of the distribution.

Before proceeding to more quantitative calculations, we remark that obviously, for a given $B$ the feedback criterion for quenching to start will be that of Eq. (2) where $\epsilon_{\gamma}$ should be replaced by $\epsilon_{\gamma \max }$. Assuming that this condition holds and that the compactness of the $\gamma$-rays is above the critical one, the production of synchrotron photons with energy $x_{\max }=b \epsilon_{\gamma \max }^{2} / 4$ is guaranteed. This means that $\gamma$-rays with energy $\epsilon_{\gamma} \gtrsim \epsilon_{\gamma \text { min,eff }}$ should interact in $\gamma \gamma$ interactions, where

$\epsilon_{\gamma \min , \mathrm{eff}}=2 / x_{\max }=8 / b \epsilon_{\gamma \max }^{2}$. 


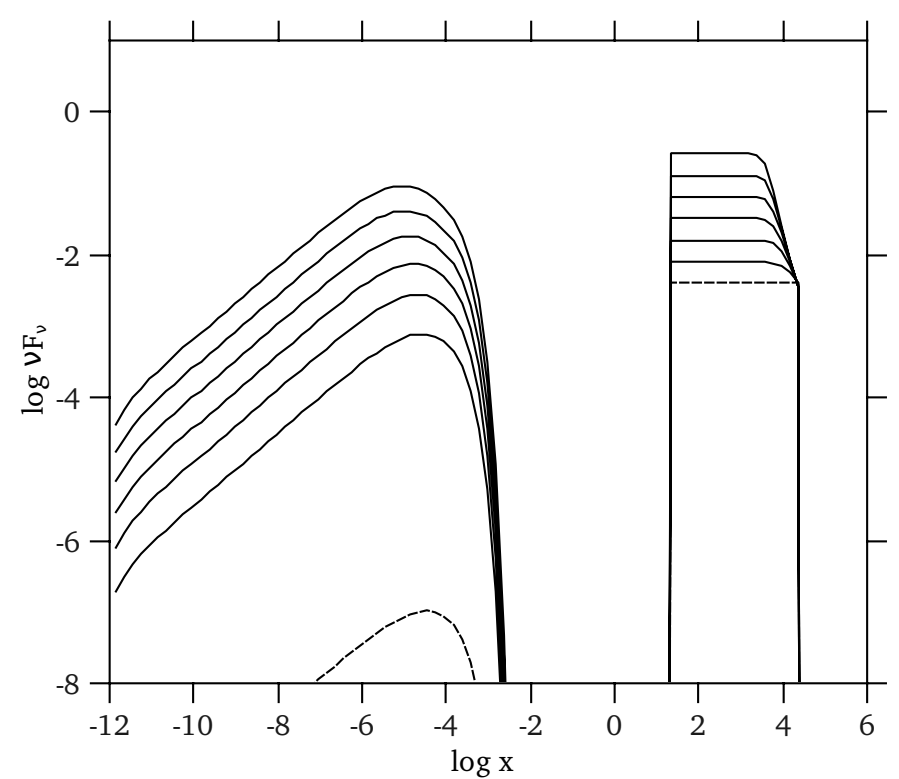

Fig. 7. MW spectra for a power-law injection of $\gamma$-rays of the form $Q_{\gamma}=$ $Q_{0} \epsilon_{\gamma}^{-2}$ for $\epsilon_{\gamma \min }<\epsilon_{\gamma}<\epsilon_{\gamma \max }$ with $\epsilon_{\gamma \min }=23$ and $\epsilon_{\gamma \max }=2.3 \times 10^{4}$ for various values of the normalization $Q_{0}$. This starts from $Q_{0}=4 \times 10^{-3}$ and then exceeds its previous value by a factor of 2 . The dashed line curves represent the runs without quenching. The source parameters are $B=40 \mathrm{G}$ and $R=3 \times 10^{16} \mathrm{~cm}$.

Thus, depending on the relation between $\epsilon_{\gamma \min , \mathrm{eff}}$ and $\epsilon_{\gamma \min }$ we can distinguish two cases:

(i) $\epsilon_{\gamma \min }<\epsilon_{\gamma \text { min,eff }}$ : in this case quenching will affect $\gamma$-rays from $\epsilon_{\gamma \max }$ down to $\epsilon_{\gamma \min , \text { eff }}$. For $\epsilon_{\gamma} \leq \epsilon_{\gamma \text { min,eff }}$ the spectrum will be unaffected, i.e., it will be the same as the one at injection.

(ii) $\epsilon_{\gamma \min , \text { eff }}<\epsilon_{\gamma \min }$ : in this case quenching will affect the whole $\gamma$-ray spectrum.

The above two cases are exemplified in Figs. 7 and 8. In both figures we assumed that $\gamma$-rays with a power-law form of slope $\alpha=2$ are injected in a source of radius $R=3 \times 10^{16} \mathrm{~cm}$ and magnetic field strength $B=40 \mathrm{G}$. The only difference between the two cases is in the lower and upper limits, which are $\epsilon_{\gamma \min }=$ 23 and $\epsilon_{\gamma \max }=2.3 \times 10^{4}$ for the case shown in Fig. 7 and $\epsilon_{\gamma \min }=$ 230 and $\epsilon_{\gamma \max }=2.3 \times 10^{5}$ for the case shown in Fig. 8 .

In the case of Fig. 7 we have $\epsilon_{\gamma \min }<\epsilon_{\gamma \text { min,eff }}=1.7 \times 10^{4}$ and, as can be seen from the figure, the power-law breaks above $\epsilon_{\gamma \text { min,eff }}$ because of quenching - indeed, we find that the value of $\epsilon_{\gamma \text { min,eff }}$ is in reality lower by a factor of 2 than the one given in Eq. (39), because in the numerical calculations shown here we use the full expressions for the synchrotron emissivity and the cross-section for $\gamma \gamma$ and not the $\delta$-function approximations implied in the derivation of the above relation. It is interesting to note that as the $\gamma$-ray luminosity increases and thus the quenching becomes more effective, the break between the unabsorbed and absorbed part of the power-law becomes more abrupt.

In the case of Fig. 8, on the other hand, the choice of the parameters were such as to make $\epsilon_{\gamma \text { min,eff }}$ comparable to $\epsilon_{\gamma \min }$. Thus, according to the qualitative analysis presented above, quenching should affect the whole $\gamma$-ray distribution. Indeed, as can be deduced from the figure, as the luminosity increases, absorption is moving progressively to lower $\gamma$-ray energies, until it affects the whole distribution.

We also investigated the relation between the slope of the injected $\left(\alpha_{\text {inj }}\right)$ and the "quenched" $\left(\alpha_{\text {que }}\right) \gamma$-ray spectrum. For that

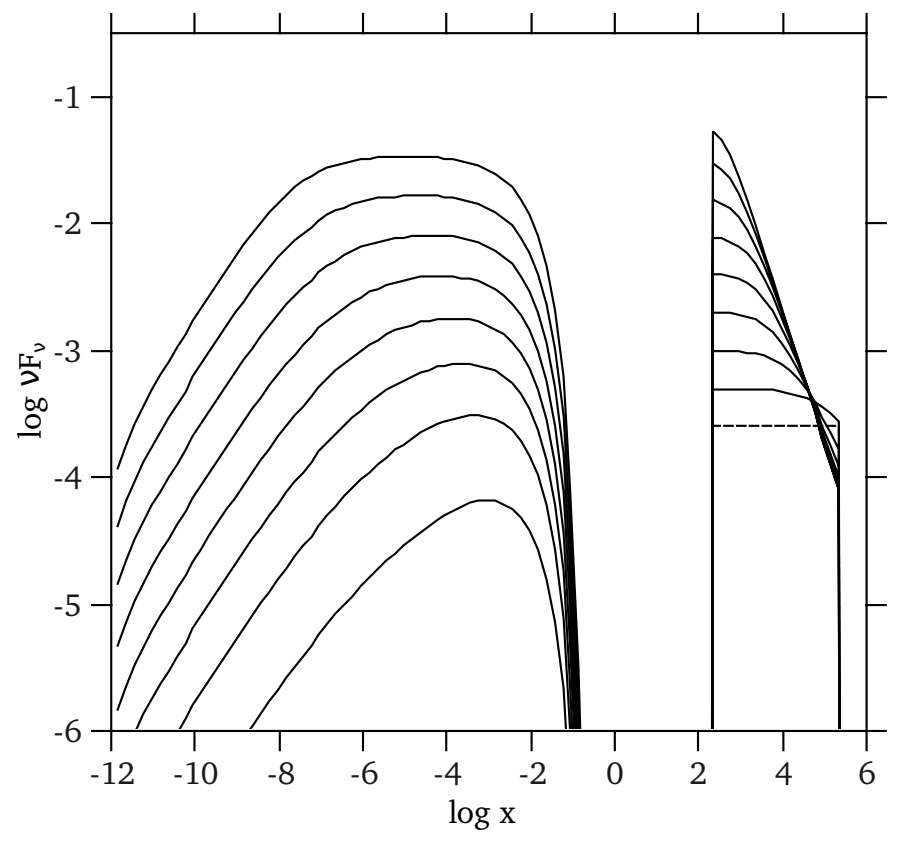

Fig. 8. Same as in Fig. 7 with $\epsilon_{\gamma \min }=2.3 \times 10^{2}$ and $\epsilon_{\gamma \max }=2.3 \times 10^{5}$ with $Q_{0}$ starting from $Q_{0}=2.5 \times 10^{-4}$.

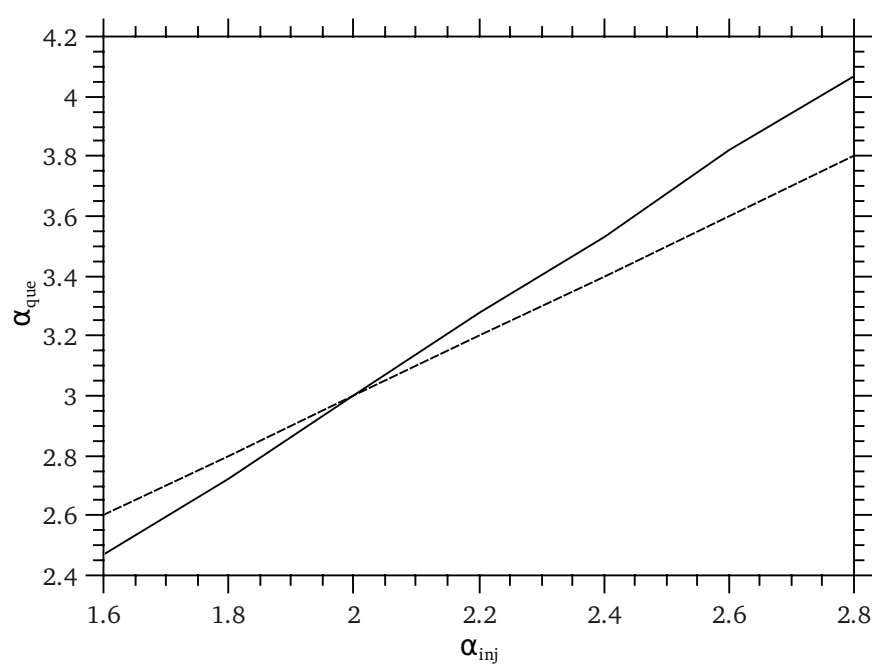

Fig. 9. Slope of the quenched $\gamma$-ray spectrum as a function of the injected spectrum's slope (solid line). To facilitate a comparison the line $\alpha_{\text {inj }}+1$ is also shown (dashed line).

reason, we ran the code for different $\alpha_{\text {inj }}$, choosing each time suitable values of the source parameters to enable quenching. Figure 9 shows the result of the runs (solid line) and the slope $\alpha_{\text {inj }}+1$ (dashed line) for comparison reasons. We find that $\alpha_{\text {que }} \propto$ $\alpha_{\text {inj }}^{1.34}$. Thus, quenching becomes more pronounced as the injected spectrum gets steeper.

\section{Application to the one-zone homogeneous models: the $3 C 279$ case}

The above has a straightforward application to the so-called onezone homogeneous models, which are customarily used to fit the MW spectra of Active Galactic Nuclei (AGN) (Böttcher et al. 2009; Aleksić et al. 2011). According to these models, the "source" contains electrons and/or protons, which radiate 
through various processes and form a spectrum that can then be fitted to the observed data by varying certain key parameters for a detailed description see Mastichiadis \& Kirk (1997) and Konopelko et al. (2003).

Alternatively, one can assume that the $\gamma$-rays are produced by some mechanism and investigate the parameter space that allows the escape of these $\gamma$-rays. To perform this analysis, one does not require the existence of other softer photons that act as targets, but one simply has to look for the threshold conditions for photon quenching. In other words, the relevant question one has to ask is: assume a spherical source of radius $R$ containing a magnetic field $B$ and producing a $\gamma$-ray spectrum that is measured at Earth with a flux $F_{\gamma}\left(\epsilon_{\gamma}\right)$. If the source is moving with a Doppler factor $\delta$ with respect to us, what is the parameter space of $R, B$, and $\delta$ that allows the $\gamma$-rays to escape? As we will show, this can give very robust lower limits for the Doppler factor $\delta$.

The luminous quasar 3C 279 is a good example. A recent comprehensive review of observations can be found in Böttcher et al. (2009). Here we will mainly focus on the 2006 campaign, which discovered the source at VHE $\gamma$-rays, showing a high TeV flux (MAGIC Collaboration et al. 2008), while the $\mathrm{X}$-rays were at a much lower level.

To apply the ideas discussed in the previous section, we follow the procedure below.

We assume a spherical source of radius $R$ moving with Doppler factor $\delta$ with respect to us and containing a magnetic field of strength $B$. We assume that $\gamma$-rays are produced by some unspecified mechanism inside the source and, upon escape, they form the observed $\gamma$-ray spectrum. If $\ell_{\gamma}^{\text {inj }}$ is the compactness of the $\gamma$-rays (cf. Eq. (1)), we can relate it to the observed integrated flux $F_{\gamma}^{\text {int }}$ by the relation

$\ell_{\gamma}^{\text {inj }}=\frac{3 \sigma_{\mathrm{T}} F_{\gamma}^{\text {int }} D_{\mathrm{L}}^{2}}{\delta^{4} R m_{\mathrm{e}} c^{3}}$

where $D_{\mathrm{L}}=3.08 \mathrm{Gpc}$ is the luminosity distance to the source. Here we used a cosmology with $\Omega_{\mathrm{m}}=0.3, \Omega_{\Lambda}=0.7$ and $H_{0}=$ $70 \mathrm{~km} \mathrm{~s}^{-1} \mathrm{Mpc}^{-1}$.

To model the $\mathrm{TeV}$ emission, we performed a $\chi^{2}$ fit to the MAGIC data, corrected for intergalactic $\gamma \gamma$ absorption (Böttcher et al. 2009), of the form $v F_{v}=A v^{-\alpha}$ for $v_{\min }^{\mathrm{obs}}<v<v_{\max }^{\mathrm{obs}}$ where $A=1.3 \times 10^{31} \mathrm{Jy} \mathrm{Hz}, \alpha=0.7, v_{\min }^{\text {obs }}=2 \times 10^{25} \mathrm{~Hz}$ and $v_{\max }^{\text {obs }}=$ $10^{26} \mathrm{~Hz}$. Then we injected $\gamma$-rays in the code of the form $Q_{\gamma}=$ $Q_{0} \epsilon_{\gamma}^{-\alpha}$ for $\epsilon_{\gamma \min }<\epsilon_{\gamma}<\epsilon_{\gamma \max }$ where the normalization is set by condition (37) with the integration taking place between $\epsilon_{\gamma \min }=$ $h v_{\min }^{\mathrm{obs}} /\left(\delta m_{\mathrm{e}} c^{2}\right)$ and $\epsilon_{\gamma \max }=h v_{\max }^{\mathrm{obs}} /\left(\delta m_{\mathrm{e}} c^{2}\right)$.

Figure 10 depicts the resulting MW spectra for $R=10^{16} \mathrm{~cm}$, $B=40 \mathrm{G}$ and $\delta$ varying between $10^{0.8}$ to $10^{1.4}$ with increments of 0.2 in the exponent. Only the run with the highest value of $\delta$ can fit the TeV data. The other three, even though they have parameters that could also fit the data, they do not because of quenching. It is important to repeat at this point that the $\gamma$-ray luminosity produced in these cases is the maximum possible that the system can radiate for the particular choice of $R, B$, and $\delta$. Any effort to increase the $\gamma$-ray flux by e.g. increasing $\ell_{\gamma}^{\text {inj }}$ would only result in an increase of the reprocessed radiation.

Figure 11 depicts a series of similar runs with $R=10^{16} \mathrm{~cm}$, $\delta=10^{1.2}$ and $B=.16 \mathrm{G}$ (solid line), $B=1.6 \mathrm{G}$ (dashed line) and $B=16 \mathrm{G}$ (dotted line). While for low $B$ values there is no quenching, this begins to appear at higher values of the magnetic field. Note, however, that the value of $B=1.6 \mathrm{G}$ produces an acceptable fit, because the soft reprocessed radiation does not violate any observable limit (most notably the X-rays)

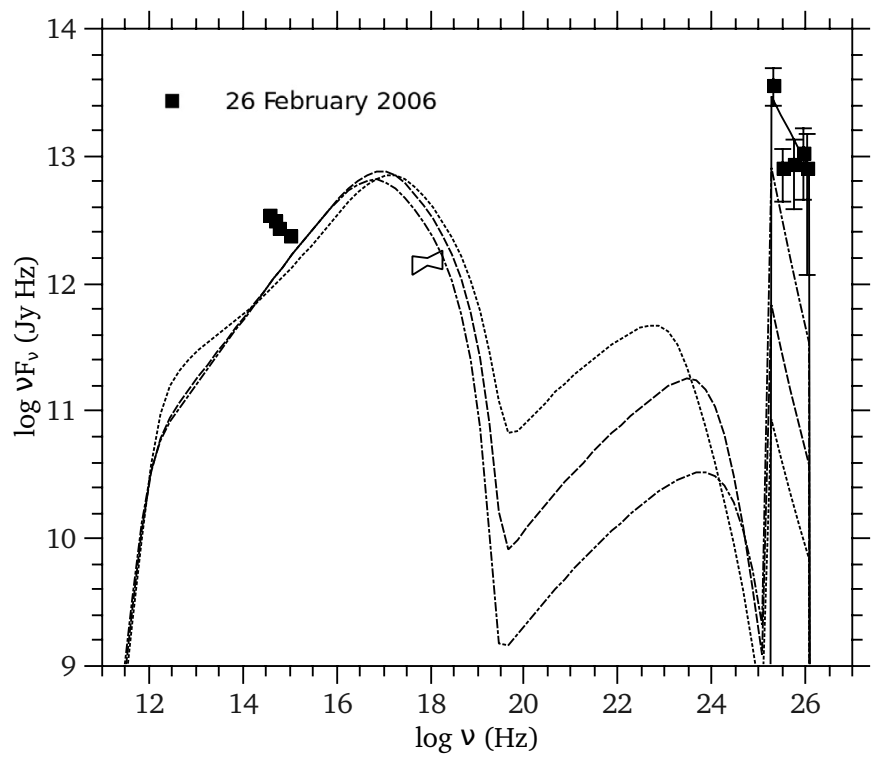

Fig. 10. MW spectra of $3 \mathrm{C} 279$ for $R=10^{16} \mathrm{~cm}, B=40 \mathrm{G}$ and $\delta=10^{0.8}$ (dotted line), $10^{1.0}$ (dashed line), $10^{1.2}$ (dot-dashed line) and $10^{1.4}$ (solid line). In all cases the $\gamma$-ray injection would have produced the solid line in the absence of quenching. Black squares and bowtie represent the observational data from February 2006.

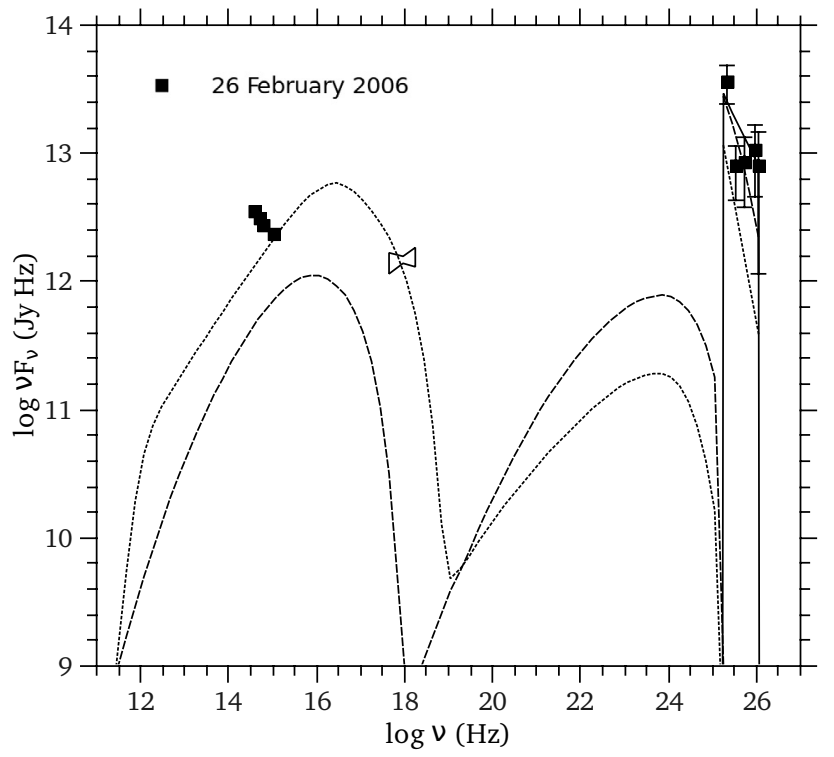

Fig. 11. MW spectra of 3C 279 for $R=10^{16} \mathrm{~cm}, \delta=10^{1.2}$ and $B=$ $.16 \mathrm{G}$ (solid line), $1.6 \mathrm{G}$ (dashed line) and $16 \mathrm{G}$ (dotted line). In all cases the $\gamma$-ray injection would have produced the solid line in the absence of quenching. Black squares and bowtie represent the observational data from February 2006.

and it can produce a marginally acceptable fit to $\mathrm{TeV} \gamma$-rays. Based on this, one can define a limiting value of the magnetic field $B_{\mathrm{q}, \mathrm{mx}}(R, \delta)$ with the property that if $B>B_{\mathrm{q}, \mathrm{mx}}$, the quenching does not allow the internally produced $\gamma$-ray luminosity to escape. Therefore $B_{\mathrm{q}, \mathrm{mx}}$ is the highest allowed value that the $B$ field can take for the observed flux of TeV $\gamma$-rays.

Figure 12 depicts the locus $B_{\mathrm{q}, \mathrm{mx}}$ as a function of the size of the source $R$ for various Doppler factors $\delta$. Each line separates the parameter space into two regions: because of the quenching, a choice of $R$ and $B$ above the locus produces a far too low $\gamma$-ray 


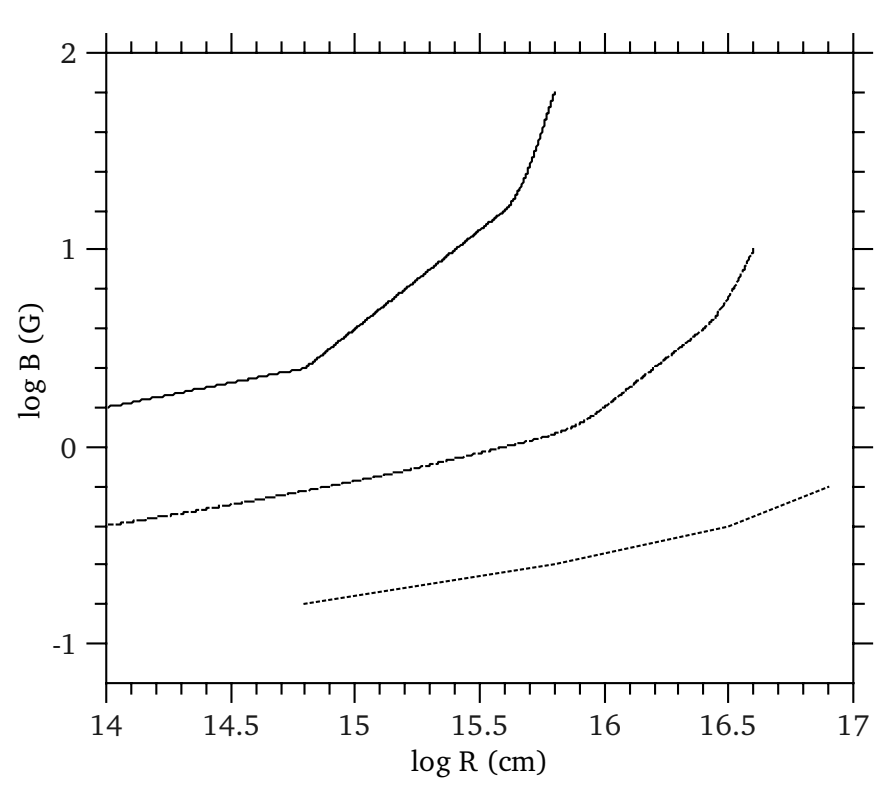

Fig. 12. Parameter space of the allowed values of the magnetic field $B$ as a function of the size of the source $R$ for Doppler factors of $\delta=10$ (dotted line), 15.8 (dashed line), and 25 (solid line). The space above a line for a certain $\delta$ corresponds to combinations of $R$ and $B$ that cannot successfully fit the $\mathrm{TeV}$ observations of $3 \mathrm{C} 279$.

flux that cannot fit the TeV observations. For example, if one assumes that $\delta=10$ and $R=2.5 \times 10^{16} \mathrm{~cm}-$ so that it corresponds to a variability timescale of 1 day, the magnetic field cannot exceed $0.3 \mathrm{G}$. However, owing to the steep dependence of the flux on $\delta$ (cf. Eq. (40)), the above condition is greatly relaxed for higher values of $\delta$.

We should also mention that $B_{\mathrm{q}, \mathrm{mx}}$ not only depends on $\delta$ and $R$, but also on the upper cutoff $\epsilon_{\gamma \max }$ of the injected power-law in $\gamma$-rays. For the calculations performed here, we chose $\epsilon_{\gamma \max }$ such as to match the highest observed frequency in the data set, i.e., $10^{26} \mathrm{~Hz}$. If one assumes that the internally produced $\gamma$-rays can extend to even higher energies, then the value of $B_{\mathrm{q}, \mathrm{mx}}$ is affected as well. Accordingly we found that if we were to repeat the same calculations with $\epsilon_{\gamma \max }$ ten times higher than the value we used, then quenching would affect the flux more and the lines of Fig. 12 would have to shift upwards by a factor of 2 . Therefore, Fig. 12 can be taken as a conservative lower limit on $B_{\mathrm{q}, \mathrm{mx}}$.

\section{Summary and discussion}

We have examined the effects of photon quenching on compact $\gamma$-ray sources. Automatic photon quenching, first proposed by $\mathrm{SK}$, is a non-linear network of two processes that are very common in high-energy astrophysics, namely photon-photon annihilation and lepton synchrotron radiation. We verified and expanded both analytically and numerically the results of SK and we showed that this network, when applied to compact $\gamma$-ray sources, can give limits on the Doppler factor, which depend on the source radius and on the magnetic field strength only. This is a theoretically derived limit and as such does not depend on the soft photon populations that might be present inside the source.

It is important to note that photon quenching can be investigated only when the radiation transfer problem is solved selfconsistently because it is a non-linear process that involves redistribution of energy from the $\gamma$-rays to the lower energy parts of the photon spectrum. For this we used a system of kinetic equations that treats in the simplest case $\gamma$-ray annihilation and electron synchrotron radiation. Using suitable $\delta$-function approximations for the photon-photon annihilation cross section and for the photon synchrotron emissivity, we were able to verify the results of SK in the limit where synchrotron losses can be considered as catastrophic (see Fig. 1). On the other hand, using a more realistic step function for the cross section, we were able to find a very good agreement with numerical results, which utilized the full photon-photon annihilation cross section. Furthermore, we extended our numerical study in the case where $\gamma$-rays are injected in the form of a power-law. We found that quenching can be responsible for wide spectral breaks, which become more pronounced as the injected $\gamma$-rays become steeper (see Fig. 9).

Quenching has some interesting consequences when applied to existing $\gamma$-ray sources. For example, for the 2006 MAGIC observations of the quasar $3 \mathrm{C} 279$, modelling with combinations of low values of the Doppler factor $\delta$ and high values of the magnetic field strength $B$ lead to strong absorption of the injected $\gamma$-rays. The only way to avoid quenching is either to assume typical values of $\delta$ (i.e., around 10) and low values of $B$ or, alternatively, high values for both $\delta$ and $B$ - see Fig. 12 .

We restricted our analysis to cases where $\gamma$-rays are injected into the source without being too specific in assigning a particular radiation mechanism for producing them. We preferred this approach because our aim was to calculate the effects of quenching using simple functional forms for the $\gamma$-rays and not to model the source in detail. For example, we could have used proton synchrotron radiation to fit the $3 \mathrm{C} 279 \mathrm{TeV}$ observations. However, this would have involved extra parameters for the radiating relativistic protons and would have complicated the analysis beyond the level at which we would like to present it here.

In our analytical treatment we ignored inverse Compton scattering, because its inclusion would have complicated the analysis. On the other hand, it was taken into account in all our numerical calculations, although it is greatly suppressed by Klein-Nishina effects (see Fig. 5). This process can still make an impact in cases where the soft photon compactness greatly exceeds the magnetic one (cf. Eq. (14)). However, this has to be seen in conjunction with the overall fitting of the MW spectrum of the source and we avoided presenting it here, although we took it into account in Sect. 4 when performing the analysis on quasar 3C 279.

Concluding, quenching has many far-reaching implications for the modelling of compact $\gamma$-ray sources such as Active Galactic Nuclei and Gamma Ray Bursts. Because quenching operates in an autoregulatory manner by coupling electrons to photons, its effects on the photon spectra cannot be manifested if the modelling is done by assuming an ad-hoc radiating electron and/or proton distribution. This could lead to parameter choices that lie within the quenching "forbidden" parameter space, making them in essence invalid.

On the other hand, if taken fully into account, quenching can give robust limits for important source parameters such as its size, magnetic field, and the Doppler factor for a given $\gamma$-ray flux. In the case of 3C 279 for example, we found that to avoid quenching, the source should have a large Doppler factor $(\delta \gtrsim 10)$ whenever high $B$ values are adopted. Similar constraints could be found for other AGNs with spectra extending to even higher energies $(\sim 1-10 \mathrm{TeV})$. In these cases the conditions for quenching are more stringent, because they critically depend on the maximum energy of the $\gamma$-ray photons and are certainly worth investigating further. 
A\&A 532, A11 (2011)

Acknowledgements. We would like to thank Dr. John Kirk for useful comments and discussion. This research has been co-financed by the European Union (European Social Fund - ESF) and Greek national funds through the operational Program "Education and Lifelong Learning" of NSRF - Research Funding Program: Heracleitus II.

\section{References}

Aleksić, J., Antonelli, L. A., Antoranz, P., et al. 2011, A\&A, 530, A4 Bonometto, S., \& Rees, M. J. 1971, MNRAS, 152, 21

Böttcher, M. 2007, Ap\&SS, 309, 95

Böttcher, M., \& Chiang, J. 2002, ApJ, 581, 127

Böttcher, M., Reimer, A., \& Marscher, A. P. 2009, ApJ, 703, 1168

Coppi, P. S. 1992, MNRAS, 258, 657

Guilbert, P. W., Fabian, A. C., \& Rees, M. J. 1983, MNRAS, 205, 593

Herterich, K. 1974, Nature, 250, 311
Jelley, J. V. 1966, Nature, 211, 472

Kataoka, J., Takahashi, T., Makino, F., et al. 2000, ApJ, 528, 243

Katarzyński, K., Ghisellini, G., Tavecchio, F., et al. 2005, A\&A, 433, 479

Kazanas, D. 1984, ApJ, 287, 112

Kirk, J. G., \& Mastichiadis, A. 1992, Nature, 360, 135

Konopelko, A., Mastichiadis, A., Kirk, J., de Jager, O. C., \& Stecker, F. W. 2003, ApJ, 597, 851

MAGIC Collaboration, Albert, J., Aliu, E., Anderhub, H., et al. 2008, Science, 320,1752

Mastichiadis, A., \& Kirk, J. G. 1995, A\&A, 295, 613

Mastichiadis, A., \& Kirk, J. G. 1997, A\&A, 320, 19

Petropoulou, M., \& Mastichiadis, A. 2009, A\&A, 507, 599

Stawarz, Ł., \& Kirk, J. G. 2007, ApJ, 661, L17

Stern, B. E., Begelman, M. C., Sikora, M., \& Svensson, R. 1995, MNRAS, 272, 291

Svensson, R. 1987, MNRAS, 227, 403

Zdziarski, A. A., \& Lightman, A. P. 1985, ApJ, 294, L79 